\title{
Finer-Scale Extraction of Viscoelastic Properties from Nanoindentation Characterised by Viscoelastic-Plastic Response
}

\author{
A. Jäger* and R. Lackner ${ }^{\dagger}$ \\ *Institute for Mechanics of Materials and Structures, Vienna University of Technology, Vienna, Austria \\ †Material-Technology Unit, University of Innsbruck, Technikerstraße 13, 6020 Innsbruck, Austria
}

\begin{abstract}
Motivated by recent progress in viscoelastic indentation analysis, the identification of viscoelastic properties from materials exhibiting elastic, viscous and plastic material behaviour by means of nanoindentation is dealt with in this paper. Based on existing solutions for pure viscoelastic material behaviour, two methods allowing us to consider the effect of plastic deformation are presented: (i) the so-called double-indentation technique, with the second indentation characterised by pure viscoelastic material response and (ii) the use of spherical indenter geometries instead of commonly used pyramidal indenters avoiding plastic deformation at all. Both methods are applied to three different polymers, giving access to the model parameters of the fractional dash-pot which is used to describe the viscoelastic behaviour. The results obtained are compared with results from standard (single) indentation tests using a Berkovich indenter. Moreover, the influence of the maximum load, determining the amount of plastic material response, on the identified model parameters is investigated. Finally, the creep-compliance functions identified by nanoindentation are compared with the respective macroscopic creep-compliance functions obtained from bendingbeam rheometer tests.
\end{abstract}

KEY WORDS: creep, double indentation, nanoindentation, parameter identification, viscoelasticity

\section{Introduction}

Recent progress in both finer-scale experimentation (atomic force microscopy, nanoindentation, etc.) and theoretical and numerical upscaling schemes provides the basis for the development of so-called multiscale models, taking finer scales of observation for describing and predicting macroscopic material behaviour into account. The success of multiscale models is strongly linked to the proper identification of material properties at finer scales, serving as input for the upscaling schemes mentioned. In this paper, extraction of viscoelastic material parameters by means of the nanoindentation technique is presented. Parameter identification of materials exhibiting only elastic and time-dependent material response is based on the measured increase of penetration during the so-called holding phase of the measured penetration history [1-13] (see Figure 1A). Hereby, experimental data are compared with the respective data from analytical solution for the mathematical problem of a rigid indenter penetrating a viscoelastic medium [14-22], giving access to the parameters of the underlying viscoelastic model. Such back-analysis schemes, how- ever, are only applicable to materials showing no plastic behaviour. In case of plastic deformation occurring during the loading phase of nanoindentation tests, both plastic and viscoelastic deformations would be assigned to the viscoelastic material response, leading to wrong parameters for the underlying viscoelastic model overestimating the viscoelastic material response [7, 23-25]. In order to account for the effect of plastic deformation, two methods are compared in this paper: (i) the double-indentation technique and (ii) use of spherical indenter geometries instead of the commonly used pyramidal indenters.

As regards the double-indentation technique (see the succeeding section), the effect of plastic deformation is determined by adapting the experimental setup (change of load history) and considered in the employed parameter-identification scheme. The motivation for using spherical indenter geometries, on the other hand, is to avoid the development of plastic deformation by reducing the stresses under the indenter during the indentation process. Therefore, the experimental setup used for a single indentation (trapezoidal load history) may be employed when using spherical indenters and the parameter 
(A)

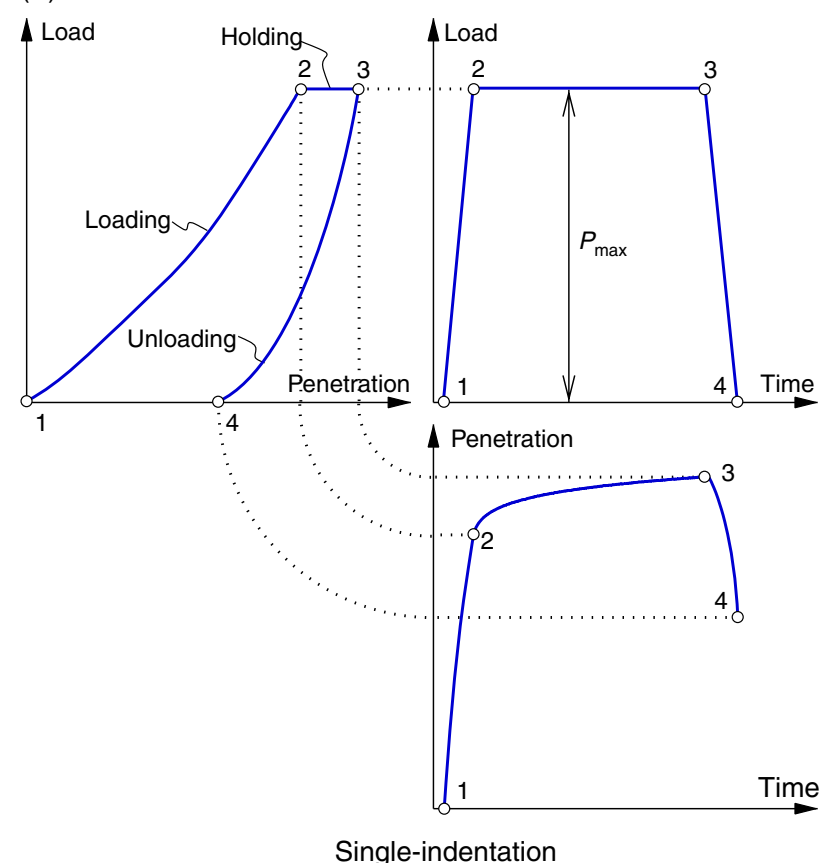

(B)

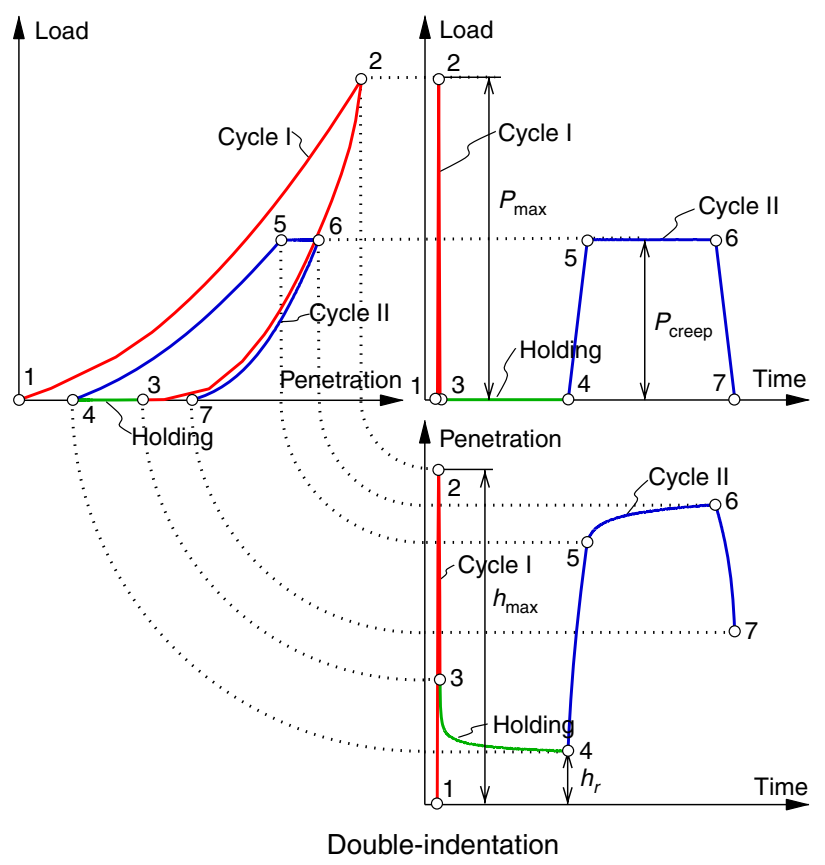

Figure I: Load-penetration curve, load history and penetration history for (A) single-indentation test and (B) double-indentation test on material exhibiting elastic, viscous and plastic material response

identification scheme must be extended towards spherical tip geometries (see section Parameter Identification of Viscoelastic Properties). In order to assess the performance of the two methods presented, tests on three polymers are conducted using either a Berkovich or a cono-spherical tip and employing both the single- as well as the double-indentation method (see section Materials and Experimental Setup). The model parameters identified from the experimental data are presented and discussed in the Results and Discussion section. Concluding remarks are given in the final section.

\section{Single Versus Double Indentation}

Recently, pre-loading of the material was performed by Zhang et al. [25], leading to the so-called doubleindentation technique (see Figure 1B). Hereby, the load history is characterised by two load cycles, with the first cycle (pre-loading) leading to plastic deformation and the second cycle showing only viscoelastic deformation. Thus, the latter is suitable for back calculation of viscoelastic properties using analytical solutions for the respective viscoelastic indentation problem. The second load cycle is characterised by the penetration of a tip into the imprint resulting from the first load cycle. In the elastic case, this reloading cycle may be described by the contact problem of two conforming solids [26-29]. More refined models, considering, e.g. the shape of the plastic imprint, were presented in [30-32]. In this paper, the concept of effective indenters as proposed by Sakai [32] is employed. According to Sakai [32], the reloading indentation process (second cycle) into a plastic imprint (stemming from the first cycle) can be approximated by the indentation of a tip with an effective tip shape into a flat undeformed surface. Hereby, the face angle $\beta$ of the original pyramidal indenter is replaced by an effective face angle $\beta_{\text {eff }}=$ $\beta-\beta_{\mathrm{r}}$, with $\tan \beta_{\mathrm{r}}=h_{\mathrm{r}} / h_{\mathrm{c}} \tan \beta$. Hereby, $h_{\mathrm{r}}$ is the residual penetration after the first load cycle and $h_{\mathrm{c}}$ is the contact height at maximum load of the first load cycle. Following this idea, adaptation of the tip-shape function $f(\rho)$ (see Figure 2), already used in the

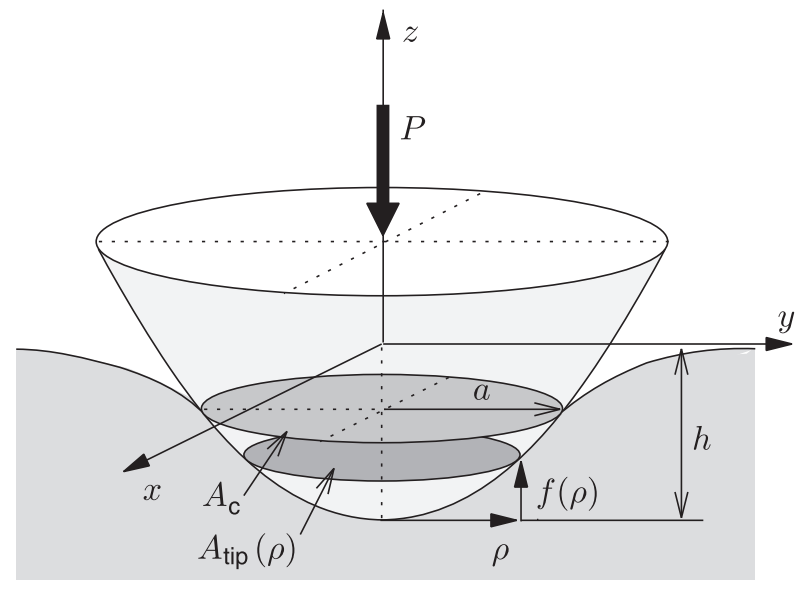

Figure 2: Contact between rigid axisymmetric indenter of shape $f(\rho)$ and an infinite half-space ( $P$ is the applied load, $h$ is the penetration and $a$ is the contact radius) 
identification scheme reported in Ref. [12], is proposed in this paper. Hereby, the effective tip shape is given as

$f_{\text {eff }}(\rho)=\left(1-\frac{h_{\mathrm{r}}}{h_{\mathrm{max}}}\right) f(\rho)$

where $h_{\mathrm{c}}$ was replaced by $h_{\max }$ (penetration at point 2 , see Figure $1 \mathrm{~B}$ ) in order to avoid the use of wrong values of $h_{\mathrm{c}}$ resulting from negative values of $S$, i.e. the initial unloading slope of the load penetration curve, occurring during testing of low viscous materials. The residual penetration $h_{\mathrm{r}}$ is the value of the penetration at point 4 (see Figure $1 \mathrm{~B}$ ), accounting for the viscoelastic deformation taking place during the holding period after unloading, reducing the residual penetration depth. The identification of model parameters is identical to the identification scheme proposed in Ref. [12], considering the holding phase of the second load cycle and the effective tip shape $f_{\text {eff }}(\rho)$.

\section{Parameter Identification of Viscoelastic Properties}

For identification of viscoelastic properties, the procedure outlined in Refs $[12,13]$ is followed. Hereby, the tip shape is approximated by the distance

$f(\rho)=\frac{1}{2 C_{0}}\left(\sqrt{C_{1}^{2}+4 C_{0} \rho^{2} \pi}-C_{1}\right)$

from the apex of the axisymmetric tip as a function of the radius $\rho(\mathrm{m})$ (see Figure 2), giving the crosssection area of the tip, $A_{\text {tip }}$, as

$A_{\text {tip }}(\rho)=\rho^{2} \pi=C_{0} f(\rho)^{2}+C_{1} f(\rho)$

Similar to the procedure proposed in Ref. [33], the tip shape is described by two constants, $C_{0}(-)$ and $C_{1}$ $(\mathrm{m})$, which are obtained from calibration of the nanoindentation-testing equipment (see section Materials and Experimental Setup). With these two constants and the function $f(\rho)$, both perfect cones with a semi-apex angle $\alpha\left(C_{0}=\pi \tan ^{2} \alpha, C_{1}=0\right)$ and perfect spheres with a tip radius $R\left(C_{0}=-\pi, C_{1}=\right.$ $2 R \pi$ ) can be described. As regards the required solution of the elastic indentation problem, the solution outlined in Ref. [12], applicable only to tip shapes characterised by $C_{0}>0$ and $C_{1}>0$, is extended to a more general solution also including the special case of spherical tip shapes $\left[C_{0}<0\right.$ and $C_{1}>0, \rho \leq \rho_{\max }$, with $\left.\rho_{\max }=\sqrt{-C_{1}^{2} /\left(4 C_{0} \pi\right)}\right]$. According to the so-called Sneddon solution [34], the relation between the penetration $h(\mathrm{~m})$ and the corresponding load $P$ $(\mathrm{N})$ is given for an axisymmetric indenter of shape $f(\rho)$ penetrating an elastic half-space by

$h=a \int_{\rho=0}^{a} \frac{f^{\prime}(\rho) \mathrm{d} \rho}{\sqrt{a^{2}-\rho^{2}}} \quad P=2 \frac{E}{1-v^{2}} \int_{\rho=0}^{a} \frac{\rho^{2} f^{\prime}(\rho) \mathrm{d} \rho}{\sqrt{a^{2}-\rho^{2}}}$.

Hereby, $a(\mathrm{~m})$ is the radius of the projected contact area $A_{\mathrm{c}}, E$ is Young's modulus, $v$ is Poisson's ratio and $f^{\prime}=\mathrm{d} f / \mathrm{d} \rho$. Inserting Equation (2) into Equation (4) gives the penetration and the applied load as a complex function of the contact radius $a$, reading

$$
\begin{array}{r}
h=\frac{1}{2 \sqrt{C_{0}}}\left(i a \sqrt { \pi } \left(\log \left[-\frac{i\left(C_{1}^{2}+4 a^{2} C_{0} \pi\right)}{\sqrt{C_{0}}}\right]\right.\right. \\
\left.\left.-\log \left[4 a C_{1} \sqrt{\pi}+\frac{i\left(-C_{1}^{2}+4 a^{2} C_{0} \pi\right)}{\sqrt{C_{0}}}\right]\right)\right), \\
P=\frac{E}{1-v^{2}} \frac{1}{8 C_{0}^{3 / 2} \sqrt{\pi}}\left(4 a C_{1} \sqrt{C_{0} \pi}-i\left(C_{1}^{2}-4 a^{2} C_{0} \pi\right)\right. \\
\left(\log \left[-\frac{i\left(C_{1}^{2}+4 a^{2} C_{0} \pi\right)}{\sqrt{C_{0}}}\right]\right. \\
\left.\left.-\log \left[4 a C_{1} \sqrt{\pi}+\frac{i\left(-C_{1}^{2}+4 a^{2} C_{0} \pi\right)}{\sqrt{C_{0}}}\right]\right)\right)=M F(a)
\end{array}
$$

where $M$ is the indentation modulus, with $M=$ $E /\left(1-v^{2}\right)$ and

$$
\begin{aligned}
F(a)= & \frac{1}{8 C_{0}^{3 / 2} \sqrt{\pi}}\left(4 a C_{1} \sqrt{C_{0} \pi}-i\left(C_{1}^{2}-4 a^{2} C_{0} \pi\right)\right. \\
& \left(\log \left[-\frac{i\left(C_{1}^{2}+4 a^{2} C_{0} \pi\right)}{\sqrt{C_{0}}}\right]\right. \\
& \left.\left.-\log \left[4 a C_{1} \sqrt{\pi}+\frac{i\left(-C_{1}^{2}+4 a^{2} C_{0} \pi\right)}{\sqrt{C_{0}}}\right]\right)\right)
\end{aligned}
$$

is a function depending only on geometrical properties, such as the tip shape (described by $C_{0}$ and $C_{1}$ ) and the contact radius $a$. It is noted, that for arbitrary values of $C_{0}$ and $C_{1}$ Equations (5) and (6) always give real numbers for $h$ and $P$. For negative values of $C_{0}$ (spherical shape), the contact radius $a$ is restricted by

$a \leq \rho_{\max }=\sqrt{\frac{-C_{1}^{2}}{4 C_{0} \pi}}$

with $\rho_{\max }$ as the maximum possible.

Based on the analytical solution of the elastic indentation problem [Equations (5) and (6)] for the tip shape given in Equation (3), the viscoelastic solution is obtained by the application of the method of functional equations [16]. Hereby, the elastic 
operators $P, M$ and $F(a)$ in Equation (6) are replaced by their Laplace transforms $\hat{P}(s), \hat{M}(s)$ and $\hat{F}(a(s))$, giving after re-arrangement

$\hat{F}(a(s))=\frac{\hat{P}(s)}{\hat{M}(s)}=\frac{1}{s \hat{M}(s)} s \hat{P}(s)=\hat{\bar{Y}}(s) s \hat{P}(s)$,

where $1 /(s \hat{M}(s))$ was replaced by the Laplace transform of $\bar{Y}(t)$, referred to as indentation compliance function in the following. Back transformation to the time domain gives

$F(a(t))=\int_{0}^{t} \bar{Y}(t-\tau) \frac{\mathrm{d} P(\tau)}{\mathrm{d} \tau} \mathrm{d} \tau$

The left-hand side of Equation (10) depends only on geometric properties, i.e. the constant parameters $C_{0}$ and $C_{1}$, and on the experimentally obtained contact radius $a(t)$. The right-hand side of Equation (10), on the other hand, depends on the mechanical properties of the viscoelastic half-space represented by the indentation compliance function $\bar{Y}(t)$ and the load history $P(t)$. The still unknown contact radius $a$ is determined from the measured penetration $h$ using Equation (5).

For identification of viscoelastic model parameters for the materials presented in the following section, a fractional dash-pot is used. The respective creepcompliance function reads

$J(t)=J_{\mathrm{a}}\left(\frac{t}{\bar{\tau}}\right)^{k}$

where $J_{\mathrm{a}}\left(\mathrm{GPa}^{-1}\right)$ is the creep compliance at $t=\bar{\tau}$ and $k(-)$ is the creep exponent, with $0 \leq k \leq 1 . \bar{\tau}$ is introduced for dimensional reasons and is set equal to $1 \mathrm{~s}$. The fractional dash-pot is able to cover a wide range of material behaviour from pure elastic $(k=0)$ to pure viscous $(k=1)$ materials. Considering the indentation compliance function for incompressible materials, with $\bar{Y}(t)=J(t) / 4$, Equation (10) becomes, for the fractional dash-pot and the holding phase of a trapezoidal load history [13],

$$
\begin{aligned}
F_{\mathrm{H}}\left(J_{\mathrm{a}}, k, t\right) & =\int_{0}^{t} \frac{J(t-\tau)}{4} \frac{\mathrm{d} P(\tau)}{\mathrm{d} \tau} \mathrm{d} \tau \\
& =\frac{P_{\max }}{4 \tau_{\mathrm{L}}}\left\{J_{\mathrm{a}} \frac{1}{k+1}\left(\frac{1}{\bar{\tau}}\right)^{k}\left[t^{k+1}-\left(t-\tau_{\mathrm{L}}\right)^{k+1}\right]\right\},
\end{aligned}
$$

where $\tau_{\mathrm{L}}(\mathrm{s})$ is the loading duration. In order to determine model parameters from nanoindentation test data, the error between the analytical model response [Equation (12)] and $F(a(t))$ obtained from the nanoindentation test data [Equation (7)], denoted as $F_{\text {exp}}$, is minimised within the holding phase by adapting the unknown model parameters $J_{\mathrm{a}}$ and $k$. The mentioned error is defined by

$R\left(J_{\mathrm{a}}, k\right)=\frac{e\left(J_{\mathrm{a}}, k\right)}{u}$,

with

$e^{2}\left(J_{\mathrm{a}}, k\right)=\sum_{i=1}^{n}\left[F_{\exp }\left\{a\left[h\left(t_{i}\right)\right]\right\}-F_{\mathrm{H}}\left(J_{\mathrm{a}}, k, t_{i}\right)\right]^{2}$,

and

$u^{2}=\sum_{i=1}^{n} F_{\exp }^{2}\left(t_{i}\right)$

with $t_{i}$ denoting different time instants within the holding phase.

As regards the double-indentation technique, the tip-shape function $f(\rho)$ is replaced by the effective tip shape $f_{\text {eff }}(\rho)=\left(1-h_{\mathrm{r}} / h_{\text {max }}\right) f(\rho)$. Considering $f_{\text {eff }}(\rho)$ in the presented identification scheme, the functions describing the tip shape given in Equations (7) and (5) are adapted, reading $F_{\text {exp,eff }}(a)=\left(1-h_{\mathrm{r}} / h_{\text {max }}\right) F_{\exp }(a)$ and $h_{\text {eff }}=\left(1-h_{\mathrm{r}} / h_{\text {max }}\right) h$. Moreover, $t=0$ refers to the beginning of the second load cycle (point 4 in Figure 1B).

\section{Materials and Experimental Setup}

Three different polymers, i.e. low-density polyethylene (LDPE), polymethyl methacrylate (PMMA) and polycarbonate (PC), are considered in this study. The respective values for Young's modulus and Poisson's ratio were taken from the literature and are listed in Table 1. Nanoindentation tests were conducted at room temperature using a Hysitron Triboindenter nanoindenter (Hysitron, Minneapolis, MN, USA) with a three-sided Berkovich diamond tip and a cono-spherical tip with a nominal tip radius of $5 \mu \mathrm{m}$.

Table I: Young's modulus and Poisson's ratio of polymers considered

\begin{tabular}{llll}
\hline Polymer (source) & LDPE (http://www.matweb.com) & PMMA (http://www.matweb.com) & PC (Bayer Industry Services) \\
\hline Young's modulus (GPa) & $0.139-0.35$ & $1.8-3.1$ & $2.3-2.4$ \\
Poisson's ratio (-) & 0.38 & $0.35-0.4$ & 0.37 \\
\hline
\end{tabular}


All tests were performed with a closed-loop feedback control, providing a constant load during the holding phase of the test. As the drift correction gave unstable results, the drift correction was de-activated for all nanoindentation tests conducted.

The load history of the single indents (see Figure $1 \mathrm{~A}$ ) is characterised by a loading phase with a duration of $3 \mathrm{~s}$ followed by a holding phase of $20 \mathrm{~s}$ at maximum load. During double indentation, the load was applied in $0.2 \mathrm{~s}$ in the first load cycle and then reduced to $1 \mu \mathrm{N}$ in $0.2 \mathrm{~s}$. After a holding phase of $20 \mathrm{~s}$ at a load of $1 \mu \mathrm{N}$, the load was increased again in $3 \mathrm{~s}$ to $50 \%$ of the load applied in the first load cycle, and held constant for $20 \mathrm{~s}$. In order to study the dependence of the identified model parameters on the load considered during the nanoindentation test, the following maximum loads were applied: (i) LDPE: 1000, $750,500,250,125,50 \mu \mathrm{N}$ for the Berkovich tip and $750,500,375,250,125,50,25 \mu \mathrm{N}$ for the spherical tip; (ii) PMMA: 10000, 7500, 5000, 2500, 1000, 500, 250, 100, $50 \mu \mathrm{N}$ for both tip shapes; (iii) PC: 10000 , 7500, 5000, 2500, 1000, $60 \mu \mathrm{N}$ for both tip shapes.

For determination of the macroscopic creep-compliance function, bending beam-rheometer tests were performed. Hereby, a specimen with the height $h$ $(\mathrm{mm})$ and the width $b(\mathrm{~mm})$ is placed horizontally on two bearings (spacing $l=102 \mathrm{~mm}$ ). During the test, a constant load $F$ is applied for $240 \mathrm{~s}$ at the midspan of the beam. The compliance function $J(t)$ is obtained from the measured increase of the mid-span deflection $u(t)$ by extending the solution for the elastic case

$u=\frac{F l^{3}}{4 b h^{3}} \frac{1}{E}=\frac{F l^{3}}{4 b h^{3}} \frac{1}{2(1+v) G}=\frac{F l^{3}}{12 b h^{3}} \frac{1}{G}$,

where an incompressible material behaviour (Poisson's ratio $v=0.5$ ) is assumed, to the case of viscoelastic deviatoric behaviour, replacing $1 / G$ by $J(t)$, reading

$J(t)=\frac{12 b h^{3}}{F l^{3}} u(t)$

\section{Tip-shape calibration}

The area function of the Berkovich tip was obtained by the standard calibration procedure outlined in Ref. [35] using fused quartz (FQ) as calibration material. For the cono-spherical tip, on the other hand, additionally single-crystal aluminium ( $\mathrm{Al}$ ) was used for the calibration, giving access to the area function for larger penetrations (up to $1.2 \mu \mathrm{m}$ ). The calibration procedure, however, is the same for both materials and tip shapes:
1 Perform indents in a material with given elastic properties (e.g. FQ) in the depth range of the indentation experiments.

2 Compute the projected contact area $A_{\mathrm{c}}=\pi / 4(S)$ $M)^{2}$, where $S\left(\mathrm{~N} \mathrm{~m}^{-1}\right)$ is the initial unloading slope of the load-penetration curve and $M$ is the indentation modulus, with $M=E /\left(1-v^{2}\right)$, where $E=72 \mathrm{GPa}$ and $v=0.17$ for FQ.

3 Plot $A_{\mathrm{c}}$ as a function of the contact depth $h_{\mathrm{c}}$, with $h_{\mathrm{c}}=h-0.75 P_{\max } / S$, and approximate the soobtained function by

$A_{\mathrm{c}}=C_{0} h_{\mathrm{c}}^{2}+C_{1} h_{\mathrm{c}}$

where $C_{0}(-)$ and $C_{1}(\mathrm{~m})$ are constants describing the tip shape.

The machine compliance, required for determination of the correct penetration depth in the calibration procedure, was determined according to Ref. [36] using the Berkovich tip.

Figure 3 shows the area function $A_{\text {tip }}(f(\rho))$ for the Berkovich and the cono-spherical tip. Although the shape of the Berkovich tip is well approximated by a cone over the whole penetration range $\left(C_{0}\right.$ and $C_{1}$ remain positive and approximately constant), the shape of the cono-spherical tip changes from spherical (Figure 3A) to conical (Figure 3B) and again to spherical (Figure 3C). These findings were also observed in scanning electron microscopy images (see Figure 4), showing the mentioned change of the tip shape from spherical, to conical, and again to spherical.

\section{Results and Discussion}

All three polymers were tested considering different values for the maximum load using the single- and double-indentation technique using the Berkovich and the cono-spherical tip. For each maximum load, at least nine indents were conducted and mean values for the identified parameters for the fractional dash-pot, $J_{\mathrm{a}}$ and $k$, were computed. Figures 5-7 show the identified model parameters as a function of the maximum penetration depth which is directly related to the respective maximum applied loads, for LDPE, PMMA and PC obtained for single and double indentation for both tip shapes.

For all four test setups (single and double indentation using a Berkovich or a cono-spherical tip) a higher variation of the creep exponent at small loads is observed. This variation is assigned to the influence of the drift during NI testing, which is more pronounced for small penetrations. 

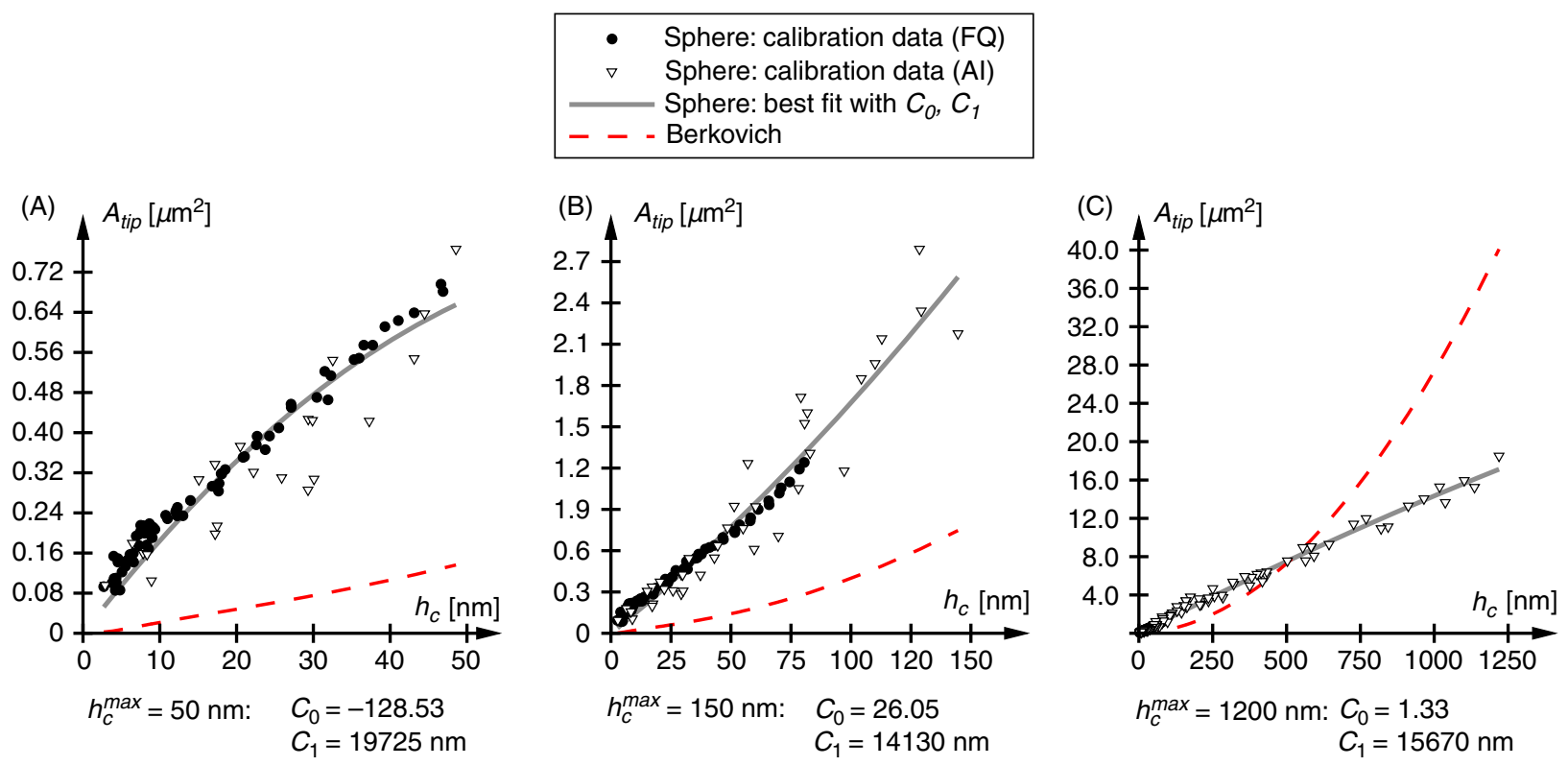

Figure 3: Area function $A_{\text {tip }}$ for Berkovich and cono-spherical tip plotted for different penetration depths (values for $C_{0}$ and $C_{1}$ refer to cono-spherical tip)

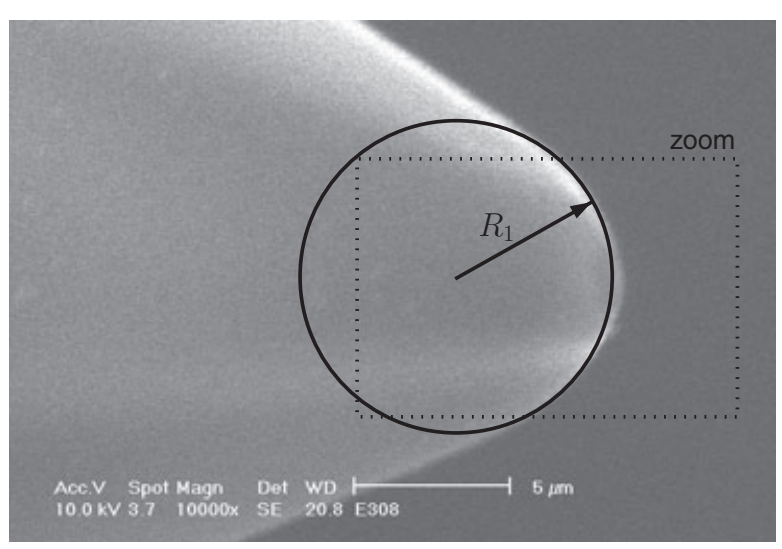

zoom

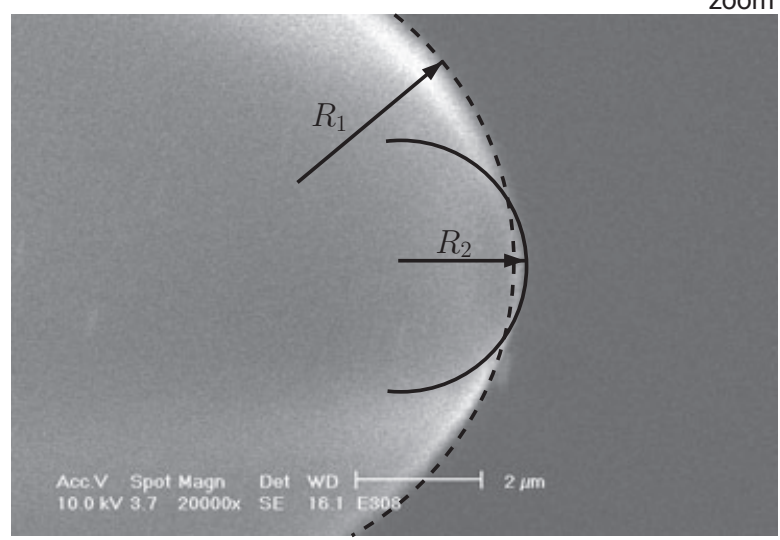

Figure 4: Scanning electron microscope image of the conospherical tip employed, showing the change of the tip shape

As regards the results from testing with the Berkovich tip, both methods reveal a similar load dependence of $J_{\mathrm{a}}$ and $k$ for all three materials, reaching a constant value of both parameters as the maximum load increases (see Table 2). Comparing the identified parameters obtained from single and double indentation, the single-indentation technique was found to overestimate the initial creep compliance for all three polymers, which is explained by the occurrence of plastic deformation. The creep exponent, on the other hand, is only slightly underestimated by the single-indentation technique.

The results obtained from testing with the conospherical tip show a quite different dependence of the identified parameters on the maximum load. For very small penetration depths, for which the tip shape is spherical (see section Tip-shape Calibration), the single- and double-indentation techniques give the same values for the model parameters. Furthermore, these values agree very well with the results from double indentation using the Berkovich tip. However, with increasing penetration depth, the model parameters obtained from single indentation using the cono-spherical tip increase continuously. This increase is explained by the larger amount of plastic deformation as the penetration depth increases. The model parameters obtained from double indentation, on the other hand, show a different behaviour with the highest value for both parameters at a minimum penetration depth of about $300 \mathrm{~nm}$. Although the initial creep compliance seems to reach a constant value for increasing penetration depth, the creep exponent increases continuously. This variation of the identified parameters is assigned to the tip shape, changing from spherical to conical and to spherical again (see Figure 3). As the employed calibration procedure for the tip shape is based on two parameters $\left(C_{0}\right.$ and $\left.C_{1}\right)$, the complicated tip shape is well approximated only for small penetration depths (within the first spherical region). In fact, 
(A)
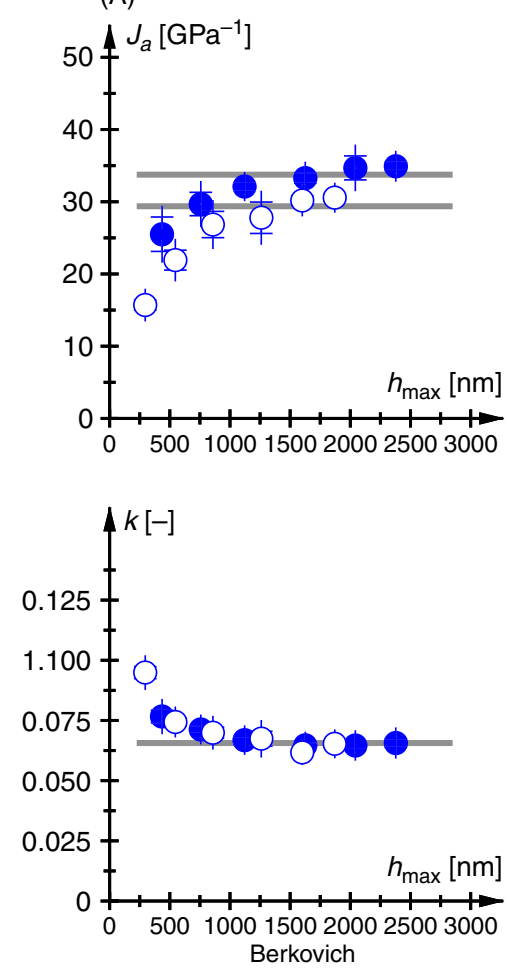

(B)
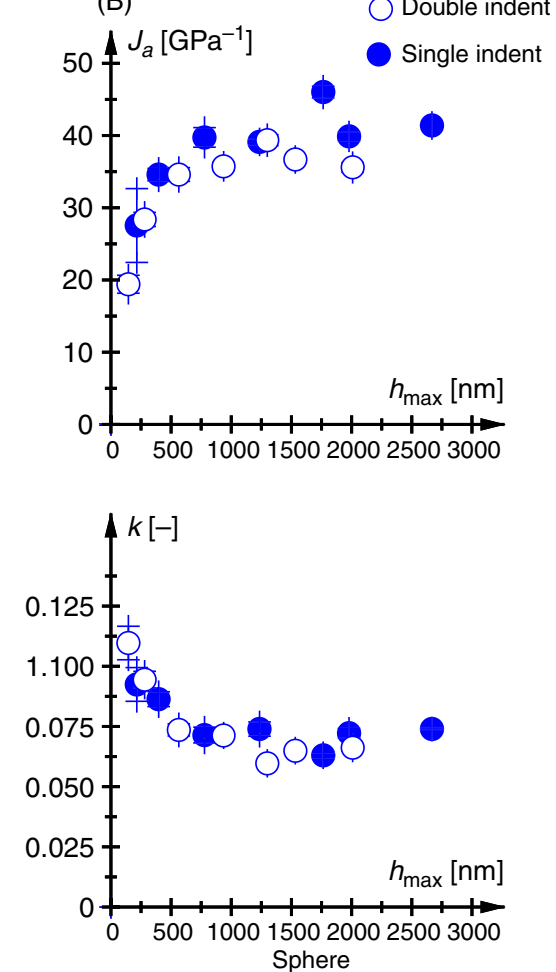

Figure 5: Fractional dash-pot parameters for LDPE obtained from single and double indentation using (A) a Berkovich tip and (B) a cono-spherical tip for different values of $h_{\max }$
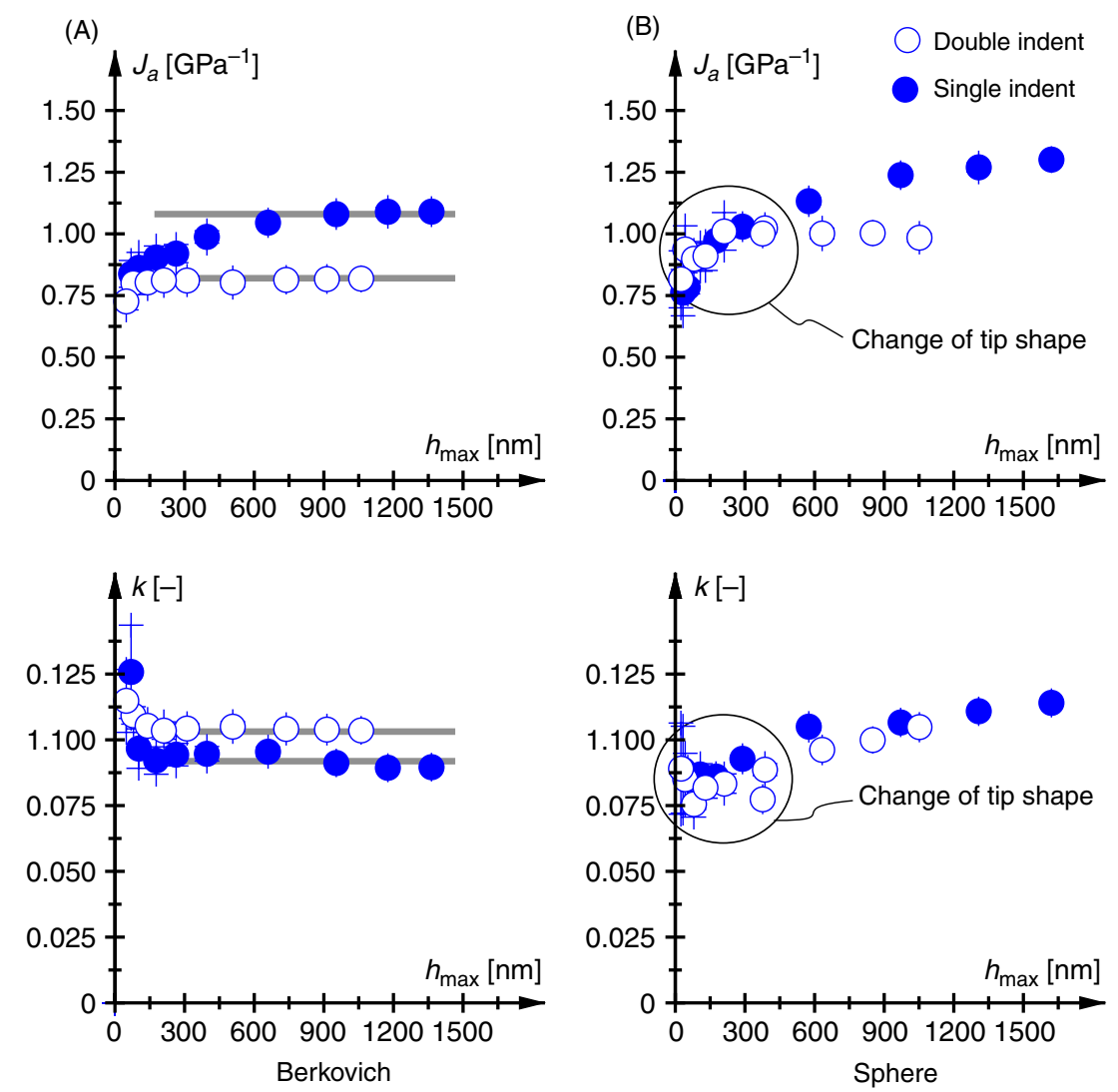

Figure 6: Fractional dash-pot parameters for PMMA obtained from single and double indentation using (A) a Berkovich tip and (B) a cono-spherical tip for different values of $h_{\max }$

for such penetration depths, both the single- and double-indentation techniques give the similar results to those obtained from double indentation using the Berkovich tip, highlighting the purely viscoelastic behaviour in case of penetration using a spherical tip. 

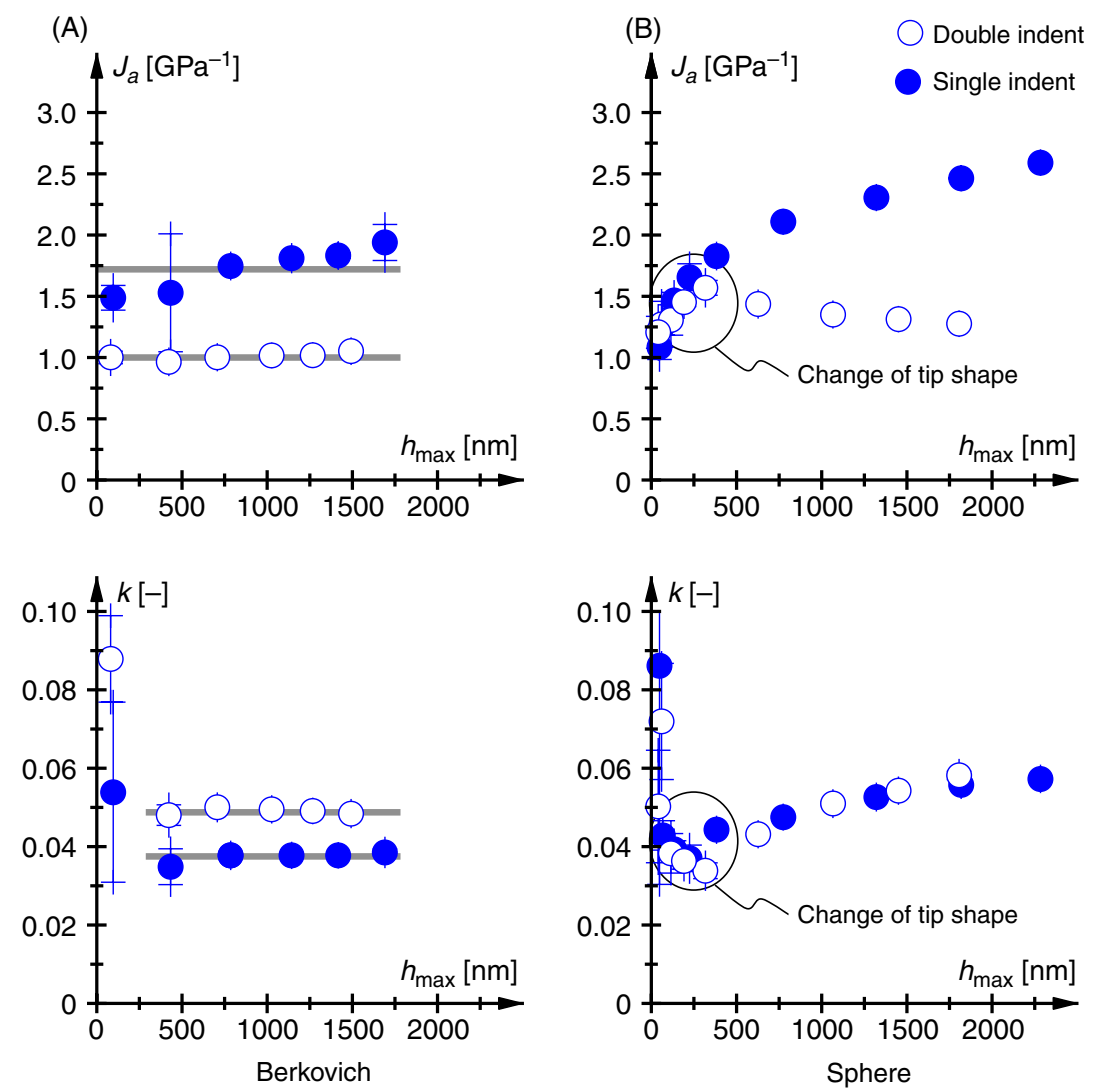

Figure 7: Fractional dash-pot parameters for PC obtained from single and double indentation using (A) a Berkovich tip and (B) a cono-spherical tip for different values of $h_{\max }$

Table 2: Initial creep compliance $J_{\mathrm{a}}$ and creep exponent $k$ for considered polymers obtained from single- and double-indentation testing using a Berkovich and a cono-spherical tip

\begin{tabular}{llllll}
\hline & Tip & Test method & LDPE & PMMA & PC \\
\hline Initial creep compliance $\int_{\mathrm{a}}\left(\mathrm{GPa}^{-1}\right)$ & Berkovich & Single indentation & 33.75 & 1.00 & 1.77 \\
& & Double indentation & 28.84 & 0.81 & 1.01 \\
& Cono-spherical & & 27.00 & 0.90 & 1.10 \\
Creep exponent $k(-)$ & Berkovich & Single indentation & 0.065 & 0.093 & 0.037 \\
& & Double indentation & 0.066 & 0.105 & 0.049 \\
& Cono-spherical & & 0.090 & 0.090 & 0.045 \\
\hline
\end{tabular}

In order to assess the identified model parameters, the creep-compliance function [Equation (11)] is plotted for all three polymers using the parameters given in Table 2 (see Figure 8) and compared with the creep-compliance function obtained from bending-beam-rheometer tests using Equation (17). In contrast to the results obtained from single indentation, the creep-compliance function corresponding to model parameters identified via double indentation shows a good agreement with the macroscopic test results for all three materials considered. Especially in case of PC, showing the highest amount of plastic deformation, the parameters identified via single indentation lead to a creep-compliance function significantly overestimating the macroscopic properties. The creep-compliance function corresponding to the model parameters identified via double indentation, on the other hand, agrees well with the compliance function obtained from the bending beam-rheometer test.

\section{Summary and Conclusions}

The identification of viscoelastic model parameters from indentation tests was dealt with in this paper. Accounting for the plastic material response during the loading phase of indentation tests, results from single and double indentation using a Berkovich and a spherical tip were presented. The parameters for the 


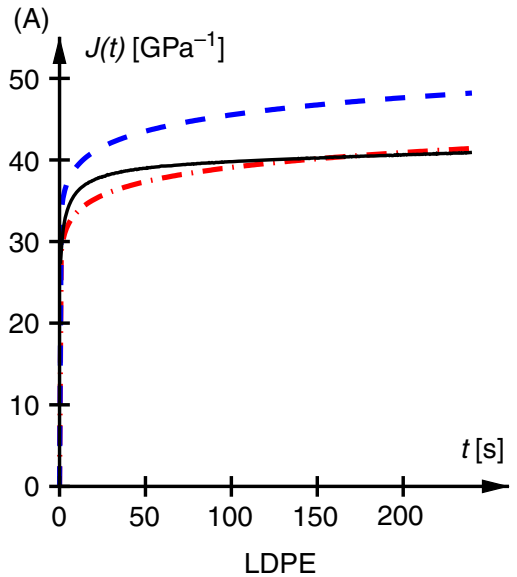

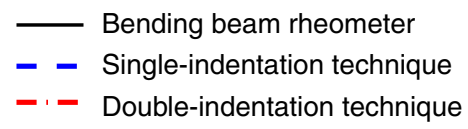

(B)

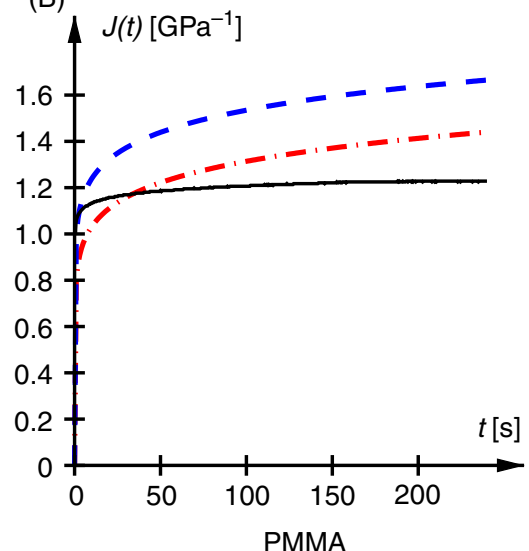

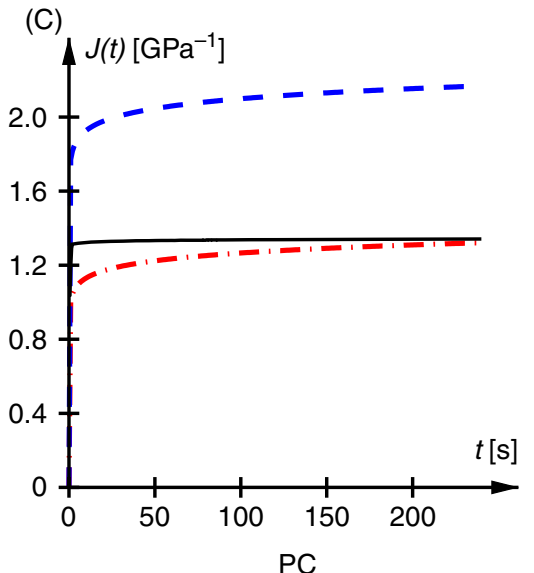

Figure 8: Creep-compliance function obtained from single- and double-indentation technique using a Berkovich tip for (A) LDPE, (B) PMMA and (C) PC compared with creep-compliance functions obtained from bending beam-rheometer tests

fractional dash-pot, describing the viscoelastic behaviour of the three types of polymers considered, were determined from back calculation using the increase of penetration during the holding phase of the indentation test. Based on the so-obtained model parameters, the following conclusions can be drawn:

1 The initial creep compliance of the fractional dash-pot identified via single indentation overestimates the creep compliance obtained from double indentation, which is explained by assigning the plastic deformation to the viscoelastic material response, giving higher values for the initial creep compliance.

2 For spherical tip shapes (within the first spherical region of the cono-spherical tip), the same parameters were identified using single and double indentation, indicating that no plastic deformation occurred during the indentation process. Furthermore, the identified parameters agreed well with results from double indentation using the Berkovich tip indicating the correct consideration of the plastic deformation in the presented double-indentation technique.

3 The parameters determined via single indentation lead to a creep-compliance function significantly overestimating the creep-compliance function obtained from bending beam-rheometer tests. The creep-compliance function obtained from the model parameters identified via double indentation using the Berkovich tip as well as using the spherical tip, on the other hand, showed good agreement with the macroscopic properties, highlighting the good performance of the presented double-indentation technique.
In summary, both the double-indentation technique as well as the use of spherical indenters were found to be appropriate for considering/avoiding plastic deformation during the identification of viscoelastic model parameters for materials exhibiting viscoelastic and plastic material behaviour. The conventional single-indentation technique using a Berkovich tip, on the other hand, may be employed in exceptional cases of materials dominated by viscoelastic material response, such as low-density polyethylene.

\section{ACKNOWLEDGEMENTS}

The authors thank all other members of the Christian Doppler Laboratory for 'Performance-Based Optimization of Flexible Pavements' at Vienna University of Technology for helpful comments and fruitful discussions on the presented research work. Financial support by the Christian Doppler Gesellschaft (Vienna, Austria) is gratefully acknowledged.

\section{REFERENCES}

1. Cheng, L., Xia, X., Yu, W., Scriven, L. E. and Gerberich, W. W. (2000) Flat-Punch indentation of viscoelastic material. J. Polymer Sci. B: Polymer Phys. 38, 10-22.

2. Cheng, L., Xia, X., Scriven, L. E. and Gerberich, W. W. (2005) Spherical-tip indentation of viscoelastic material. Mech. Mater. 37, 213-226.

3. Larrson, P.-L. and Carlsson, S. (1998) On microindentation of viscoelastic polymers. Polymer Testing 17, 49-75.

4. Sakai, M. and Shimizu, S. (2001) Indentation rheometry for glass-forming materials. J. Non-Cryst. Solids 282, 236-247.

5. Sakai, M. (2002) Time-dependent viscoelastic relation between load and penetration for an axisymmetric indenter. Phil. Mag. A 82, 1841-1849. 
6. Lu, H., Wang, B., Ma, J., Huang, G. and Viswanathan, H. (2003) Measurement of creep compliance of solid polymers by nanoindentation. Mech Time-Dependent Mater. 7, 189-207.

7. Oyen, M. L. and Cook, R. F. (2003) Load-displacement behavior during sharp indentation of viscous-elasticplastic materials. J. Mater. Res. 18, 139-150.

8. Oyen, M. L., Cook, R. F., Emerson, J. A. and Moody, N. R. (2004) Indentation response of time-dependent films on stiff substrates. J. Mater. Res. 19, 2487-2497.

9. Oyen, M. L. (2005) Spherical indentation creep following ramp loading. J. Mater. Res. 20, 2094-2100.

10. Cheng, Y.-T. and Cheng, C.-M. (2004) Scaling, dimensional analysis, and indentation measurements. Mater. Sci. Eng. R 44, 91-149.

11. Vandamme, M. and Ulm, F.-J. (2006) Viscoelastic solutions for conical indentation. Int. J. Solids Struct. 43, 31423165 .

12. Jäger, A., Lackner, R. and Eberhardsteiner, J. (2007) Identification of viscoelastic properties by means of nanoindentation taking the real tip geometry into account. Meccanica 42, 293-306.

13. Jäger, A., Lackner, R. and Stangl, K. (2007) Microscale characterization of bitumen-back-analysis of viscoelastic properties by means of nanoindentation. Int. J. Mater. Res. (formerly Z. Metallkd.) 98, 404-413.

14. Lee, E. H. (1955) Stress analysis in visco-elastic bodies. Quart. Appl. Math. 13, 183-190.

15. Radok, J. R. M. (1957) Visco-elastic stress analysis. Quart. Appl. Math. 15, 189-202.

16. Lee, E. H. and Radok, J. R. M. (1960) The contact problem for viscoelastic bodies. J. Appl. Mech. 27, 438-444.

17. Hunter, S. C. (1960) The Hertz problem for a rigid spherical indenter and a viscoelastic half-space. J. Mech. Phys. Solids 8, 219-234.

18. Graham, G. A. C. (1965) The contact problem in the linear theory of viscoelasticity. Int. J. Eng. Sci. 3, 27-46.

19. Graham, G. A. C. (1967) Contact problem in linear theory of viscoelasticity when time dependent contact area has any number of maxima and minima. Int. J. Eng. Sci. 5, 495-514.

20. Yang, W. H. (1966) Contact problem for viscoelastic bodies. J. Appl. Mech. 33, 395-401.

21. Ting, T. C. T. (1966) Contact stresses between a rigid indenter and a viscoelastic half-space. J. Appl. Mech. 33, 845-854.
22. Ting, T. C. T. (1968) Contact problems in linear theory of viscoelasticity. J. Appl. Mech. 35, 248-254.

23. Shimizu, S., Yanagimoto, T. and Sakai, M. (1999) Pyramidal indentation load-depth curve of viscoelastic materials. J. Mater. Res. 14, 4075-4086.

24. Sakai, M., Shimizu, S. and Ito, S. (2002) Viscoelastic indentation of silicate glasses. J. Am. Ceramic Soc. 85, 1210-1216.

25. Zhang, C. Y., Zhang, Y. W., Zeng, K. Y. and Shen, L. (2005) Nanoindentation of polymers with a sharp indenter. J. Mater. Res. 20, 1597-1605.

26. Steuermann, E. (1939) On Hertz theory of local deformation of compressed bodies. Comptes rendues (Doklady) de l'Academie des Sciences de l'URSS 25, 359-361.

27. Perrson, A. (1964) On the stress distribution on cylindrical elastic bodies in contact. Dissertation, Chalmers Tekniska Hogskola, Göteborg.

28. Goodman, L. E. and Keer, L. M. (1965) The contact stress problem for an elastic sphere indenting an elastic cavity. Int. J. Solids Struct. 1, 407-415.

29. Johnson, K. L. (1992) Contact Mechanics. Cambridge University Press, Cambridge.

30. Stilwell, N. A. and Tabor, D. (1961) Elastic recovery of conical indentations. Proc. Phys. Soc. 78, 169-179.

31. Pharr, G. M. and Bolshakov, A. (2002) Understanding nanoindentation unloading curves. J. Mater. Res. 17, 2660-2671.

32. Sakai, M. (2003) Elastic recovery in the unloading process of pyramidal microindentation. J. Mater. Res. 18, 16311640.

33. Thurn, J. and Cook, R. F. (2002) Simplified area function for sharp indenter tips in depth sensing indentation. J. Mater. Res. 17, 1143-1146.

34. Sneddon, I. N. (1965) The relation between load and penetration in the axisymmetric Boussinesq problem for a punch of arbitrary profile. Int. J. Eng. Sci. 3, 47-57.

35. Oliver, W. C. and Pharr, G. M. (1992) An improved technique for determining hardness and elastic modulus using load and displacement sensing indentation experiments. J. Mater. Res. 7, 1564-1583.

36. Hysitron Inc. (2006) Triboindenter User Manual. Hysitron Inc., Minneapolis, MN. 